\title{
Physicochemical properties, rheological behavior and morphology of pectin-pea protein isolate mixtures and conjugates in aqueous system and oil in water emulsion
}

\begin{abstract}
The application of pectin as an emulsifier in oil-in-water $(\mathrm{O} / \mathrm{W})$ emulsion does not guarantee its stability for a long time. In fact, pectin is not an effective emulsifier because of its hydrophilic structure. On the other hand, pea protein isolate (PPI) does not also have a proper emulsifying activity because of its hydrophobic structure. The main objective of this study was to investigate the effects of mixing and conjugation processes on the emulsifying activity and other properties of pectin and PPI in oil-in-water $(\mathrm{O} / \mathrm{W})$ emulsion and aqueous system. The conjugation variables were pectin to PPI ratio (i.e. 1:1, 2:1, 3:1, 1:2 and 1:0 w/w) and incubation time (i.e. 0, 6, 27 and $48 \mathrm{~h}$ ). The native pectin was used as a control for a comparison purpose. The current study revealed that the long incubation $\left(48 \mathrm{~h}\right.$ at $60{ }^{\circ} \mathrm{C}$ ) resulted in the formation of a strong linkage between pectin and PPI. As a result, a hybrid conjugated polymer was formed. This hybrid polymer had the lower solubility and higher emulsifying activity than the native pectin and PPI alone. After one-month storage, the emulsion containing pectin-PPI conjugate $(3: 1 \mathrm{w} / \mathrm{w}$ with $48 \mathrm{~h}$ incubation) was the most stable sample with the smallest droplet size $(1.863 \mathrm{\varepsilon m})$ among all prepared emulsions. This emulsion had the highest negative zeta-potential ( $\overline{\mathrm{l}} 49.97 \mathrm{mV})$ and stability $(83.33 \%)$ among all samples. This study revealed that mixing and conjugation of pectin and PPI led to improve the emulsifying activity of both polymers.
\end{abstract}

Keyword: Pectin; Pea protein isolate; Conjugation; Emulsifying activity; Rheological properties; Emulsion 